\title{
189. On the Bessel Kernel for a Domain
}

\author{
By Isao Higuchi \\ Suzuka College of Technology \\ (Comm. by Kinjirô KunUgi, M. J. A., May 12, 1971)
}

1. Aronszajn and Smith [2] developed the theory of Bessel potentials from the standpoints of their functional spaces and functional completions [1]. Let $P^{\alpha}\left(R^{n}\right)$ be the functional completion of $C_{0}^{\infty}\left(R^{n}\right)$ with the norm $\|u\|_{\alpha}^{2}=\int\left(1+|\xi|^{2}\right)^{\alpha}|\hat{u}(\xi)|^{2} d \xi$. They showed that $P^{\alpha}\left(R^{n}\right)$ has the reproducing kernel $G_{2 \alpha}(x-y)$ determined by

$$
G_{2 \alpha}(x)=\frac{1}{2^{(n+2 \alpha-2) / 2} \pi^{n / 2} \Gamma(\alpha)} K_{(n-2 \alpha) / 2}(|x|)|x|^{(2 \alpha-n) / 2}
$$

where $K_{(n-2 \alpha) / 2}$ is the modified Bessel function of third kind. The purpose of this paper is to consider the kernel of functional completion $P^{\alpha}(\Omega)$ of $C_{0}^{\infty}(\Omega)$ with the norm $\|u\|_{\alpha}$. Making use of the methods of general balayage and the theory of $\alpha$-harmonic functions introduced by M. Itô [6], we define the Green function $G_{2 \alpha}^{a}(x, y)$ and $\alpha$-harmonic functions in the theory of Bessel potentials. Let $E_{2 \alpha}(\Omega)$ be the class of all positive measures of finite energy with compact support contained in $\Omega, U_{2 \alpha}^{\mu}$ be the potential of $\mu \in E_{2 \alpha}(\Omega)$ in the functional space $P^{\alpha}(\Omega)$ and $G_{2 \alpha}^{\Omega} \mu$ (resp. $\breve{G}_{2 \alpha}^{\Omega} \mu$ ) be the potential of $\mu$ with respect to the kernel $G_{2 \alpha}^{\Omega}(x, y)\left(\right.$ resp. $\left.\breve{G}_{2 \alpha}^{\Omega}(x, y)=G_{2 \alpha}^{\Omega}(y, x)\right)$. We shall prove the following results.

(1) Let $\Omega$ be a domain in $R^{n}$. Then for every $\mu \in E_{2 \alpha}(\Omega)$, there exists an $\alpha$-harmonic function $H_{2 \alpha}^{\mu}(x)$ in $\Omega$ such that

$$
U_{2 \alpha}^{\mu}(x)=G_{2 \alpha}^{\Omega} \mu(x)+H_{2 \alpha}^{\mu}(x) .
$$

(2) The following conditions are equivalent:

(a) There exists a bounded domain $\Omega(\neq \emptyset)$ in $R^{n}$ such that the Green function $G_{2 \alpha}^{\Omega}(x, y)$ is the kernel of the functional space $P^{\alpha}(\Omega)$ i.e., $U_{2 \alpha}^{\mu}=G_{2 \alpha}^{\Omega} \mu$ in $P^{\alpha}(\Omega)$ for every $\mu \in E_{2 \alpha}(\Omega)$.

(b) There exist a bounded domain $\Omega$ in $R^{n}$ and a measure $\mu(\neq 0) \in E_{2 \alpha}(\Omega)$ such that $G_{2 \alpha}^{\Omega} \mu \in P^{\alpha}(\Omega)$ and $G_{2 \alpha}^{\Omega} \mu=\breve{G}_{2 \alpha}^{\Omega} \mu$ in $P^{\alpha}(\Omega)$.

(c) $0<\alpha \leqq 1$.

2. According to Aronszajn and Smith [2], we define the Bessel potentials and summarize the results obtained in [2].

Definition 1. The Bessel potential of order $2 \alpha, \alpha>0$, of a positive measure $\mu$ is defined by $G_{2 \alpha} \mu(x)=\int G_{2 \alpha}(x-y) d \mu(y)$. We denote by $E_{2 \alpha}\left(R^{n}\right)$ the class of all positive measures for which the $2 \alpha$-energy 
is finite.

$$
\|\mu\|_{2 \alpha}^{2}=\iint G_{2 \alpha}(x-y) d \mu(x) d \mu(y)=\int G_{2 \alpha} \mu d \mu
$$

( I ) The following conditions on $\mu$ are equivalent:
(a) $\mu \in E_{2 \alpha}\left(R^{n}\right)$.
(b) $G_{\alpha} \mu \in L^{2}\left(R^{n}\right)$.
(c) $G_{2 \alpha} \mu \in P^{\alpha}\left(R^{n}\right)$.

Further every function $u$ in $P^{\alpha}\left(R^{n}\right)$ is $\mu$-integrable and $\int u d \mu$ $=\left(u, G_{2 \alpha} \mu\right)_{\alpha}$.

(II) If $0<\beta \leqq \alpha$, then $\|u\|_{\beta} \leqq\|u\|_{\alpha}$. Therefore $P^{\alpha}\left(R^{n}\right) \subset P^{\beta}\left(R^{n}\right)$, $E_{2 \beta}\left(R^{n}\right) \subset E_{2 \alpha}\left(R^{n}\right)$ and $\|\mu\|_{2 \alpha} \leqq\|\mu\|_{2 \beta}$.

Definition 2. The inner and the outer capacities of order $2 \alpha$ with respect to the Bessel kernel $G_{2 \alpha}(x-y)$ are defined as usual. Every analytic set is capacitable. We denote by $\mathfrak{A}_{2 \alpha}$ the class of all sets of $2 \alpha$ capacity 0 . Then $\mathfrak{H}_{2 \alpha} \subset \mathfrak{A}_{2 \beta}$ if $0<\beta \leqq \alpha$. A property is said to hold except $\mathfrak{H}_{2 \alpha}$ (to be written exc. $\mathfrak{U}_{2 \alpha}$ ) if the set where $i \imath$ fails to hold belongs to $\mathfrak{U}_{2 \alpha}$.

Finally we summarize the properties of the kernel $G_{2 \alpha}$.

(III) $G_{2 \alpha}(x) \in L^{1}\left(R^{n}\right) . \quad \hat{G}_{2 \alpha}(\xi)=(2 \pi)^{-n / 2}\left(1+|\xi|^{2}\right)^{-\alpha}$.

(IV) $G_{\alpha+\beta}(x)=G_{\alpha} * G_{\beta}(x)$.

( V ) If $0<\alpha \leqq 1$, then $G_{2 \alpha}(x-y)$ satisfies the balayage principle. For any positive measure $\mu$ and any closed set $F$, there exists a positive measure $\mu^{\prime}$ supported by $F$ such that $G_{2 \alpha} \mu^{\prime}(x)=G_{2 \alpha} \mu(x)$ on $F$ except $\mathfrak{A}_{2 \alpha}$ and $G_{2 \alpha} \mu^{\prime}(x) \leqq G_{2 \alpha} \mu(x)$ everywhere in $R^{n}$ (see, for example, M. Kishi [8]).

3. First, we define the potentials in the functional space $P^{\alpha}(\Omega)$.

Definition 3. We denote by $(u, v)_{\alpha}$ the inner product in the Hilbert space $P^{\alpha}(\Omega)$ corresponding to the norm $\|u\|_{\alpha}$. Let $\mu \in E_{2 \alpha}(\Omega)$, then there exists a function $U_{2 \alpha}^{\mu}$ in $P^{\alpha}(\Omega)$ such that $\left(\varphi, U_{2 \alpha}^{\mu}\right)_{\alpha}=\int \varphi d \mu$ for every $\varphi \in C_{0}^{\infty}(\Omega)$. We call $U_{2 \alpha}^{\mu}$ the Bessel potential of $\mu$ in $P^{\alpha}(\Omega)$. In the following sections, we consider the kernel of $U_{2 \alpha}^{\mu}$.

4. If $0<\alpha \leqq 1, G_{2 \alpha}(x-y)$ satisfies the balayage principle by (V). But we also want to treat with the case that $\alpha>1$. Therefore we use here the methods of balayage introduced by M. Itô [6].

Theorem 1. Let $\alpha>0$ and $p$ be the integer such that $0<\alpha-p \leqq 1$. Then for any closed set $F$ and for any positive measure $\mu$ with compact support, there exists a unique system $\left\{\mu_{i}^{\prime}\right\}_{i=0}^{p}$ of positive measures supported by $F$ satisfying

$$
\begin{array}{ll}
G_{2 \alpha} \mu(x)=\sum_{i=0}^{p} G_{2(\alpha-i)} \mu_{i}^{\prime}(x) & \text { on } F \text { exc. } \mathfrak{A}_{2(\alpha-p)} \\
G_{2 \alpha} \mu(x) \geqq \sum_{i=0}^{p} G_{2(\alpha-i)} \mu_{i}^{\prime}(x) & \text { everywhere in } R^{n},
\end{array}
$$




$$
\begin{array}{ll}
G_{2 q} \mu(x)=\sum_{i=0}^{q-1} G_{2(q-i)} \mu_{i}^{\prime}(x) & \text { on } F \text { exc. } \mathfrak{A}_{2}, \\
G_{2 q} \mu(x) \geqq \sum_{i=0}^{q-1} G_{2(q-i)} \mu_{i}^{\prime}(x) & \text { everywhere in } R^{n},
\end{array}
$$

where $q$ is any integer such that $0<q \leqq p$.

Theorem 1'. Under the same assumptions in Theorem 1, there exists a unique system $\left\{\mu_{i}^{\prime \prime}\right\}_{i=0}^{p}$ of positive measures supported by $F$ satisfying

$$
\begin{aligned}
& G_{2(\alpha-p)} \mu(x)=G_{2(\alpha-p)} \mu_{0}^{\prime \prime}(x) \quad \text { on } F \text { exc. } \mathfrak{U}_{2(\alpha-p)}, \\
& G_{2(\alpha-p)} \mu(x) \geqq G_{2(\alpha-p)} \mu_{0}^{\prime \prime}(x) \quad \text { everywhere in } R^{n}, \\
& G_{2(\alpha-p+q)} \mu(x)=G_{2(\alpha-p+q)} \mu_{0}^{\prime \prime}(x)+\sum_{i=1}^{q} G_{2(q-i+1)} \mu_{i}^{\prime \prime}(x) \quad \text { on } F \text { exc. } \mathfrak{A}_{2}, \\
& G_{2(\alpha-p+q)} \mu(x) \geqq G_{2(\alpha-p+q)} \mu_{0}^{\prime \prime}(x)+\sum_{i=1}^{q} G_{2(q-i+1)} \mu_{i}^{\prime \prime}(x) \quad \text { everywhere in } R^{n},
\end{aligned}
$$
where $q$ is any integer such that $1<q \leqq p$.

The proofs of the above theorems are the same as in [6]. The essential parts are based on the decomposition $G_{2 \alpha}=G_{2(\alpha-p)} * G_{2} * G_{2} * \cdots * G_{2}$ of $G_{2 \alpha}$.

Definition 4. According to M. Itô [6], we call $\left\{\mu_{i}^{\prime}\right\}_{i=0}^{p}\left(\operatorname{resp} .\left\{\mu_{i}^{\prime \prime}\right\}_{i=0}^{p}\right)$ the system of balayaged measures of $\mu$ on $F$ with respect to the system $\left(G_{2}, \cdots, G_{2 p}, G_{2 \alpha}\right)$ (resp. $\left.\left(G_{2(\alpha-p)}, G_{2(\alpha-p+1)}, \cdots, G_{2 \alpha}\right)\right)$. We denote by $\left\{\left(\varepsilon_{y, C \Omega}^{\prime}\right)_{i}\right\}_{i=0}^{p}$ and $\left\{\left(\varepsilon_{y, C \Omega}^{\prime \prime}\right)_{i}\right\}_{i=0}^{p}$ the systems of balayaged measures on $C \Omega$ of the unit measure $\varepsilon_{y}$ on $y$. The Green function of order $2 \alpha$ in $\Omega$ is defined by $G_{2 \alpha}^{\Omega}(x, y)=G_{2 \alpha}(x-y)-\sum_{i=0}^{p} G_{2(\alpha-i)}\left(\varepsilon_{y, C \Omega}^{\prime}\right)_{i}(x)$. If $0<\alpha \leqq 1$, then $G_{2 \alpha}^{\Omega}(x, y)=G_{2 \alpha}^{\Omega}(y, x)$ and hence $G_{2 \alpha}^{\Omega}(x, y)$ is measurable as a function of $y$.

Remark. In general $G_{2 \alpha}^{\Omega}(x, y)$ is not symmetric, but we can prove by the same way as in [6], that

$$
G_{2 \alpha}^{\Omega}(x, y)=\int \cdots \int G_{2(\alpha-p)}^{\Omega}\left(x, z_{1}\right) G_{2}^{\Omega}\left(z_{1}, z_{2}\right) \cdots G_{2}^{\Omega}\left(z_{p}, y\right) d z_{1} \cdots d z_{p}
$$

where $G_{2(\alpha-p)}^{\Omega}$ (resp. $\left.G_{2}^{\Omega}\right)$ is the Green function of order $2(\alpha-p)$ (resp. 2) for $\Omega$ and hence $G_{2 \alpha}^{\Omega}(x, y)$ is measurable as a function of $y$. For any positive measure $\mu$, put

$$
G_{2 \alpha}^{\Omega} \mu(x)=\int G_{2 \alpha}^{\Omega}(x, y) d \mu(y) \text { and } \check{G}_{2 \alpha}^{\Omega} \mu(x)=\int G_{2 \alpha}^{\Omega}(y, x) d \mu(y) .
$$

Then we have

$$
\begin{aligned}
& G_{2 \alpha}^{a} \mu(x)=G_{2 \alpha} \mu(x)-\sum_{i=0}^{p} G_{2(\alpha-i)} \mu_{i}^{\prime}(x) \\
& \check{G}_{2 \alpha}^{\Omega} \mu(x)=G_{2 \alpha} \mu(x)-G_{2 \alpha} \mu_{0}^{\prime \prime}(x)-\sum_{i=1}^{p} G_{2(\alpha-i+1)} \mu_{i}^{\prime \prime}(x) .
\end{aligned}
$$

5. First, we treat with the case that $0<\alpha \leqq 1$.

Theorem 2. Let $0<\alpha \leqq 1$ and $\Omega$ be a domain in $R^{n}$. Then the Green function $G_{2 \alpha}^{\Omega}(x, y)$ is the kernel of the functional space $P^{\alpha}(\Omega)$, that is, $U_{2 \alpha}^{\mu}=G_{2 \alpha}^{\Omega} \mu$ in $P^{\alpha}(\Omega)$ for every measure $\mu \in E_{2 \alpha}(\Omega)$. 
Proof. Let $\omega$ be a relatively compact domain such that $\bar{\omega} \subset \Omega$ and $e(x)$ be a positive function in $C_{0}^{\infty}\left(R^{n}\right)$ satisfying $e(x)=0$ for $|x| \geqq 1$ and $\int e(x) d x=1$. Put $e_{\rho}(x)=\rho^{-n} e(x / \rho)$ for $0<\rho \leqq 1$. Then

$$
\begin{aligned}
& G_{2 \alpha}^{\omega} \mu * e_{\rho}(x) \in C_{0}^{\infty}\left(R^{n}\right) \cap P^{\alpha}\left(R^{n}\right), \\
& G_{2 \alpha}^{\omega} \mu * e_{\rho}(x) \rightarrow G_{2 \alpha}^{\omega} \mu(x) \text { in } P^{\alpha}\left(R^{n}\right) \text { as } \rho \rightarrow 0,
\end{aligned}
$$

because $G_{2 \alpha}^{\omega} \mu \in P^{\alpha}\left(R^{n}\right)$ (see [2], p. 423). On the other hand

$G_{2 \alpha}^{\omega} \mu(x)=G_{2 \alpha} \mu(x)-G_{2 \alpha} \mu_{C \omega}^{\prime}(x)=0$ on $C \omega$ exc. $\mathfrak{A}_{2 \alpha}$.

Therefore, for any sufficiently small $\rho, G_{2 \alpha}^{\omega} * e_{\rho}(x) \in P^{\alpha}(\Omega)$ and hence $G_{2 \alpha}^{\omega} \mu \in P^{\alpha}(\Omega)$. We shall show that $G_{2 \alpha}^{\Omega} \mu \in P^{\alpha}(\Omega)$. Let $\left\{\omega_{n}\right\}$ be an exhaustion of $\Omega$. Then $G_{2 \alpha}^{\omega_{n}} \mu \in P^{\alpha}(\Omega)$. The sequence $\left\{\mu_{C \omega_{n}}^{\prime}\right\}$ of balayaged measures of $\mu$ on $C \omega_{n}$ converges strongly to $\mu_{C \Omega}^{\prime}$ in $E_{2 \alpha}\left(R^{n}\right)$ (cf. [3]). Therefore $\left\{G_{2 \alpha}^{\omega_{n}} \mu\right\}$ converges strongly to $G_{2 \alpha}^{\Omega} \mu$ in $P^{\alpha}\left(R^{n}\right)$ and hence $G_{2 \alpha}^{\Omega} \mu \in P^{\alpha}(\Omega)$. For any function $\varphi$ in $C_{0}^{\infty}(\Omega)$, we have

$$
\left(\varphi, U_{2 \alpha}^{\mu}\right)_{\alpha}=\int \varphi d \mu=\int \varphi d \mu-\int \varphi d \mu_{C \Omega}^{\prime}=\left(\varphi, G_{2 \alpha}^{\Omega} \mu\right)_{\alpha} .
$$

This implies that $G_{2 \alpha}^{\Omega} \mu=U_{2 \alpha}^{\mu}$ in $P^{\alpha}(\Omega)$, because $C_{0}^{\infty}(\Omega)$ is dense in $P^{\alpha}(\Omega)$.

6. Since $\hat{G}_{2 \alpha}(\xi)=(2 \pi)^{-(n / 2)}\left(1+|\xi|^{2}\right)^{-\alpha}$, there exists a distribution $T_{\alpha}$ such that $T_{\alpha} * G_{2 \alpha}=\varepsilon_{0}$ (i.e., $\left.\hat{T}_{\alpha}=(2 \pi)^{n / 2}\left(1+|\xi|^{2}\right)^{\alpha}\right)$. Similarly as in [6], we define $\alpha$-harmonic functions as follows.

Definition 5. A function $u(x)$ is said to be $\alpha$-harmonic in $\Omega$ in the theory of Bessel potentials if it satisfies the following conditions:

(a) $u(x)$ is defined in $R^{n}$ exc. $\mathfrak{H}_{2 \alpha}$ and locally integrable in $R^{n}$.

(b) $T_{\alpha} * u$ is defined and $T_{\alpha} * u=0$ in $\Omega$ in the sense of distributions.

Lemma 1. Let $\mu$ be a positive measure with finite total mass. Then the potential $G_{2(\alpha-i)} \mu(x)$ is $\alpha$-harmonic in $C s_{\mu}$ for any integer $i$ such that $0 \leqq i \leqq p$.

Proof. The equality $\left(\varepsilon_{0}-\Delta\right)^{i} G_{2 \alpha}=G_{2(\alpha-i)}$ holds for every positive integer $i$ and $\left(\varepsilon_{0}-\Delta\right)^{i} * \mu=\left(\varepsilon_{0}-\Delta\right)^{i} * T_{\alpha} * G_{2 \alpha} \mu=T_{\alpha} * G_{2(\alpha-i)} \mu$. Hence the convolution $T_{\alpha} * G_{2(\alpha-i)} \mu$ is defined. Since $\left(\varepsilon_{0}-\Delta\right)^{i} * \mu=0$ in $C s_{\mu}$, $T_{\alpha} * G_{2(\alpha-i)} \mu=0$ in $C s_{\mu}$. Therefore $G_{2(\alpha-i)} \mu(x)$ is $\alpha$-harmonic in $C s_{\mu}$.

Remark. By this lemma, the Green function $G_{2 \alpha}^{a}(x, y)$ is $\alpha$-harmonic in $\Omega-\{x\}$.

Theorem 3. Let $\Omega$ be a domain in $R^{n}$ and $\mu \in E_{2 \alpha}(\Omega)$. Then there exists an $\alpha$-harmonic function $H_{2 \alpha}^{\mu}(x)$ in $\Omega$ such that

$$
U_{2 \alpha}^{\mu}(x)=G_{2 \alpha}^{\Omega} \mu(x)+H_{2 \alpha}^{\mu}(x) .
$$

Proof. Put $H_{2 \alpha}^{\mu}=U_{2 \alpha}^{\mu}-G_{2 \alpha}^{\Omega} \mu$. Then we have

$$
H_{2 \alpha}^{\mu}=\left(U_{2 \alpha}^{\mu}-G_{2 \alpha} \mu\right)+\sum_{i=0}^{p} G_{2(\alpha-i)} \mu_{i}^{\prime} .
$$

Since $S_{\mu}$ is compact, $\int d \mu_{i}^{\prime}<+\infty$ for every $i$ such that $0 \leqq i \leqq p$. Therefore, by Lemma $1, \sum_{i=0}^{p} G_{2(\alpha-i)} \mu_{i}^{\prime}$ is $\alpha$-harmonic in $\Omega . \quad$ On the other hand, 
the convolution $T_{\alpha} *\left(U_{2 \alpha}^{\mu}-G_{2 \alpha} \mu\right)$ is defined. By the definition of $U_{2 \alpha}^{\mu}$, there exists a sequence $\left\{\mu_{n}\right\}$ of signed measures supported by $C \Omega$ such that $G_{2 \alpha} \mu_{n} \rightarrow\left(U_{2 \alpha}^{\mu}-G_{2 \alpha} \mu\right)$ strongly in $P^{\alpha}\left(R^{n}\right)$ and hence in the sense of distributions. Since $G_{2 \alpha} \mu_{n}$ is $\alpha$-harmonic in $\Omega, T_{\alpha} * G_{2 \alpha} \mu_{n}=0$ in $\Omega$ and hence $T_{\alpha} *\left(U_{2 \alpha}^{\mu}-G_{2 \alpha} \mu\right)=0$ in $\Omega$ in the sense of distributions. Therefore $\left(U_{2 \alpha}^{\mu}-G_{2 \alpha} \mu\right)$ is $\alpha$-harmonic in $\Omega$. This means that $H_{2 \alpha}^{\mu}$ is $\alpha$-harmonic in $\Omega$.

Lemma 2. Suppose $U_{2 \alpha}^{\mu}=G_{2 \alpha}^{\Omega} \mu$ for every $\mu \in E_{2 \alpha}(\Omega)$. Then $\check{G}_{2 \alpha}^{\Omega} \mu$ $=G_{2 \alpha}^{\Omega} \mu$ exc. $\quad \mathfrak{A}_{2 \alpha}$ for every $\mu \in E_{2 \alpha}(\Omega)$.

Proof. Let $\mu$ and $\nu$ be any measures in $E_{2 \alpha}(\Omega)$. By the assumption

$$
\iint G_{2 \alpha}^{\Omega}(x, y) d \nu(x) d \mu(y)=\left(U_{2 \alpha}^{\mu}, U_{2 \alpha}^{\nu}\right)_{\alpha}=\left(U_{2 \alpha}^{\nu}, U_{2 \alpha}^{\mu}\right)_{\alpha}=\iint G_{2 \alpha}^{\Omega}(y, x) d \mu(y) d \nu(x)
$$

and hence

$$
\int\left(G_{2 \alpha}^{\Omega} \mu(x)-\check{G}_{2 \alpha}^{\Omega} \mu(x)\right) d \nu(x)=0 .
$$

This means that $G_{2 \alpha}^{\Omega} \mu(x)=\breve{G}_{2 \alpha}^{\Omega} \mu(x)$ exc. $\mathfrak{A}_{2 \alpha}$, because $\nu$ is arbitrary.

Theorem 4. The following conditions are equivalent:

(a) There exists a bounded domain $\Omega(\neq \emptyset)$ in $R^{n}$ such that the Green function $G_{2 \alpha}^{\Omega}(x, y)$ is the kernel of $P^{\alpha}(\Omega)$ i.e., $U_{2 \alpha}^{\mu}=G_{2 \alpha}^{\Omega} \mu$ in $P^{\alpha}(\Omega)$ for every $\mu \in E_{2 \alpha}(\Omega)$.

(b) There exist a bounded domain $\Omega$ in $R^{n}$ and a measure $\mu \neq 0$ in $E_{2 \alpha}(\Omega)$ such that $G_{2 \alpha}^{\Omega} \mu \in P^{\alpha}(\Omega)$ and $G_{2 \alpha}^{\Omega} \mu=\check{G}_{2 \alpha}^{\Omega} \mu$ in $P^{\alpha}(\Omega)$.

(c) $0<\alpha \leqq 1$.

Remark. If there exists a domain which satisfies the condition in (a) or in (b), then by Theorem 2, every domain satisfies the same condition.

Proof. We have already showed the relations (c) $\Rightarrow$ (a)(in Theorem 2) and $(a) \Rightarrow(b)$ (in Lemma 2$)$ and therefore we shall prove here the relation $(b) \Rightarrow(c)$.

First we suppose that $1<\alpha<2$ (i.e., $p=1$ ). (b) implies

$$
G_{2 \alpha} \mu-G_{2 \alpha} \mu_{0}^{\prime}-G_{2(\alpha-1)} \mu_{1}^{\prime}=G_{2 \alpha} \mu-G_{2 \alpha} \mu_{0}^{\prime \prime}-G_{2} \mu_{1}^{\prime \prime}
$$

By Lemma 1 , this means that $G_{2} \mu_{1}^{\prime \prime}$ is $\alpha$-harmonic in $\Omega$ and hence

$$
T_{\alpha-1} * \mu_{1}^{\prime \prime}=T_{\alpha-1} * T_{1} * G_{2} \mu_{1}^{\prime \prime}=T_{\alpha} * G_{2} \mu_{1}^{\prime \prime}=0 \text { in } \Omega .
$$

By the definition of $T_{\alpha-1}$, we have $\hat{T}_{\alpha-1}=(2 \pi)^{n / 2}\left(1+|\xi|^{2}\right)^{\alpha-1}$. If $1<\alpha<2$, then by Levy-Khintchine's theorem on negative definite functions, there exists a symmetric positive measure $\sigma$ in $R^{n}-\{0\}$ such that

$$
\begin{gathered}
\left(1+|\xi|^{2}\right)^{\alpha-1}=1+\int_{|y|>0}\left(1-e^{i \xi \cdot y}\right) d \sigma(y) \\
\int_{|x|>r} d \sigma(y)<+\infty \text { and } \int_{0<|y|<r}|y|^{2} d \sigma(y)<+\infty \text { for any } r>0
\end{gathered}
$$

(see, for example, M. Itô [5]). We can see that $\int_{|y|<\delta}|y|^{2} d \sigma(y)>0$ for 
any $\delta>0$. Because, if $\int_{|y|<\delta_{0}}|y|^{2} d \sigma(y)=0$, then by (2) $\int_{|y|>0} d \sigma(y)<+\infty$. Therefore $\left(1+|\xi|^{2}\right)^{\alpha-1}$ should be bounded by (1) and hence a contradiction. Let $\varphi \in C_{0}^{\infty}(\Omega)$. Then we have

$$
0=T_{\alpha-1} * \mu_{1}^{\prime \prime}(\varphi)=\int \varphi d \mu_{1}^{\prime \prime}+\int\left(\mu_{1}^{\prime \prime}(\varphi)-\mu_{1}^{\prime \prime} * \varphi(y)\right) d \sigma(y)
$$

and hence $\int \mu_{1}^{\prime \prime} * \varphi(y) d \sigma(y)=0$. On the other hand, the fact $\int_{|y|<\delta}|y|^{2} d \sigma(y)>0$ implies that $\int \mu_{1}^{\prime \prime} * \varphi(y) \neq 0$ for a suitable choice of $\varphi$. This leads us to a contradiction.

Next we suppose that $\alpha \geqq 2$. (b) means that $G_{2 \alpha} \mu-G_{2 \alpha} \mu_{0}^{\prime \prime}$ $-\sum_{i=1}^{p} G_{2(p-i+1)} \mu_{i}^{\prime \prime}$ belongs to $P^{\alpha}(\Omega) \subset P^{2}(\Omega)$ and hence $G_{2} *\left(\sum_{i=1}^{p-1} G_{2(p-i)} \mu_{i}^{\prime \prime}+\mu_{p}^{\prime \prime}\right)$ belongs to $P^{2}\left(R^{n}\right)$. Therefore by (I) $\sum_{i=1}^{p-1} G_{2(p-i)} \mu_{i}^{\prime \prime}+\mu_{p}^{\prime \prime}$ is a function in $L^{2}\left(R^{n}\right)$. This is a contradiction, because $\mu_{p}^{\prime \prime}$ is a measure on the boundary $\partial \Omega$ of $\Omega$.

Therefore (b) implies (c). This completes the proof.

\section{References}

[1] N. Aronszajn and K. T. Smith: Functional spaces and functional completion. Ann. Inst. Fourier, 6, 125-185 (1956).

[ 2 ] - Theory of Bessel potentials, Part I. Ann. Inst. Fourier, 11, 385-475 (1961).

[3] B. Fuglede: On the theory of potentials in locally compact spaces. Acta Math., 103, 139-215 (1960).

[ 4 ] M. Itô: On $\alpha$-harmonic functions. Nagoya Math. J., 26, 205-221 (1966).

[5] — - Characterization of supports of balayaged measures. Nagoya Math. J., 28, 203-230 (1966).

[6] - Etude des potentiels d'ordre $\alpha$ et des fonctions $\alpha$-harmoniques. Inventiones Math., 8, 55-68 (1969).

[ 7 ] — : Sur le balayage relatif aux noyaux composés (to appear).

[8] M. Kishi: Positive kernels and potentials. Lecture note, Nagoya Univ. (1970-1971). 